\title{
Regarding a sudden, severe depressive episode after a stroke affecting the left cingulate gyrus: a review of post-stroke depression
}

${ }^{1}$ Psychiatry Resident; ${ }^{2}$ Attending Psychiatrist

Department of Psychiatry and Mental Health - Setúbal Hospital Centre, Setúbal, Portugal

\section{POST-STROKE DEPRESSION}

\section{Definition and Epidemiology}

Post-stroke depression (PSD) is defined as depression occurring in the context of a clinically apparent stroke, with a similar symptom profile as the so-called "primary" depression ${ }^{2,3}$. PSD is the most common psychiatric complication of cerebrovascular lesions ${ }^{7,11}$, with an overall incidence at 1 year of $11 \%{ }^{9}$ and a total estimated prevalence of $30-35 \%{ }^{2,12}$.

\section{Risk Factors}
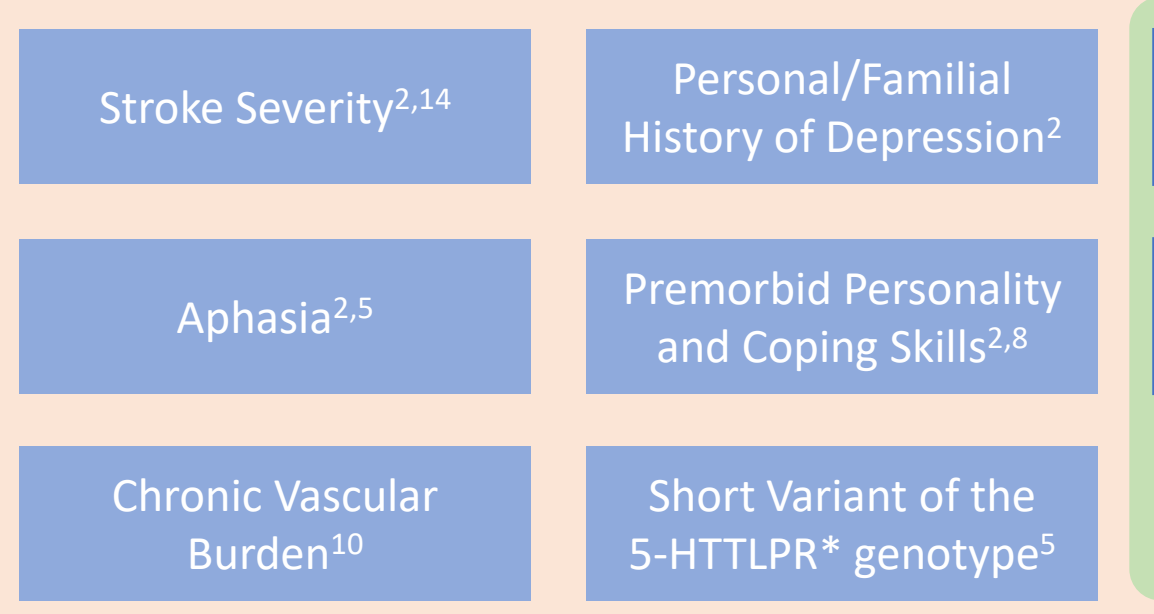

Older $\mathrm{Age}^{14}$

Female Gender ${ }^{1-3,9}$

CONTROVERSIAL

*5-HTTLPR - Serotonin Transporter Gene-linked Promoter Region

\section{Etiology}

PSD pathophysiology is still debated ${ }^{2}$. The most consistent finding in the literature is the association of PSD with stroke severity and the degree of physical and cognitive impairment ${ }^{1,5,9}$. The influence of lesion location on the development of PSD has been advocated for years by several authors, although the results are not consistent $5,6,8$. PSD has been associated with stroke lesions affecting the left cerebral hemisphere (specially the left frontal lobe and the left basal ganglia) ${ }^{3,6}$, with some authors linking it to infarcts in the territory of the anterior cerebral artery ${ }^{1}$. The frontalsubcortical circuits (FSC), connecting the thalamus and basal ganglia to the dorsolateral prefrontal cortex, the orbitofrontal cortex and the anterior cingulate cortex, seem to play a role in emotional regulation ${ }^{1}$ and lesions disrupting these networks have been implicated in $P S D^{1,2,5-8,10}$, particularly regarding the left hemisphere ${ }^{6}$. One study identified FSC infarcts as an independent radiological risk factor and a powerful predictor of $P \mathrm{SD}^{1}$.

\section{Prognosis and Treatment}

PSD is associated with prolonged recovery ${ }^{3}$, worst rehabilitation outcome $^{2,5,9}$, higher disability and cognitive impairment ${ }^{9,11}$ and higher mortality ${ }^{9}$. Antidepressants, specially Selective Serotonin Reuptake Inhibitors (SSRIs), are the drug treatment of choice ${ }^{2,3}$, as they appear not only to reduce mortality and improve functional rehabilitation in these patients ${ }^{3,4}$, but also to reduce morbidity and increase survival in both depressed and non-depressed patients ${ }^{3,13}$.

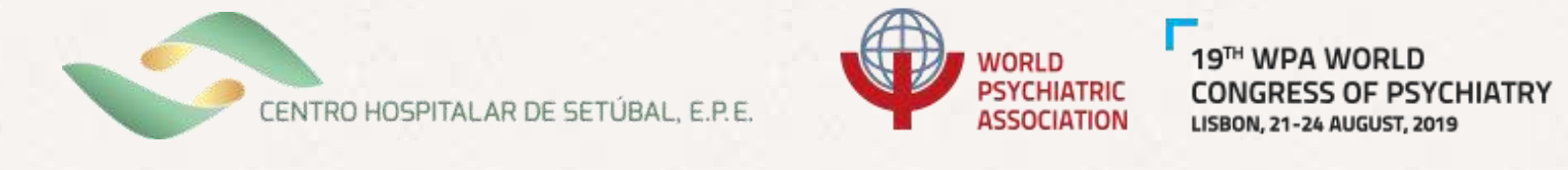

\section{CASE REPORT}

An 82-year-old man was admitted to the Psychiatric Emergency Department with depressed mood, insomnia and structured suicidal ideation, having made 2 suicide attempts in the span of 1 week. The symptoms arose rapidly 7 days prior, after a sudden onset of mild right hemiparesis, right paraesthesia and aphasia, and were not present beforehand. His relatives described him as a joyful person, a "man of success" who "was never unhappy", and the patient had no prior personal or familial psychiatric history, although he mentioned his father had killed himself after he suffered a debilitating accident when he was 80 years old. A brain CT scan was obtained, revealing an infarct in the anterior portion of the left cingulate gyrus and focal hypodensities within the left superior frontal gyrus. The patient was diagnosed with Depressive Disorder Due To Medical Condition and was admitted to the Psychiatry Ward, where he was treated with sertraline $100 \mathrm{mg} /$ day and quetiapine $50 \mathrm{mg} /$ day and began physical rehabilitation. He was discharged within 28 days, after full remission of psychiatric symptoms and improvement of post-stroke physical sequelae.

\section{DISCUSSION}

This case stands out among others described in the literature because of its sudden onset and rapid progression. Our patient was not prone to depression, had never been depressed in his life and had no prior personal or familial history of mental illness, although it should be noted his father had killed himself in a similar context (because of acquired disabilities). Despite the apparent minor severity of the stroke, the occurrence of aphasia is an important risk factor for PSD and may have predisposed our patient to PSD development. Although small, the infarct affected the anterior cingulate gyrus, which is one of the key regions of the FSC. A disruption of these networks, alongside a vascular burden along the prefrontal cortex, may serve as an explanation for such a rapid and severe case of depression after stroke in a patient with no prior history. In fact, a study showed the anterior cingulate cortex was one of the cerebral regions individually associated with $\mathrm{PSD}^{6}$. Also of importance, the lesions detected by CT scan on our patient all concerned the left hemisphere, in line with previous findings ${ }^{3,6}$. One can not rule out the psychosocial impact the physical sequelae may have had on our patient, a "man of success" who was suddenly left with disabilities in locomotion and speech. Early treatment with an SSRI improved our patient's depressive symptoms, in line with the available data ${ }^{3,8}$. We hope to highlight the need for screening for depressive symptoms in stroke patients, specially those with lesions affecting the FSC, and the positive impact of early treatment of PSD on post-stroke rehabilitation outcome and survival. 